\title{
Systematic review and meta-analysis of traditional Chinese medicine compound in treating infertility caused by endometriosis
}

\author{
Panpan Dong, Lin Ling, Lanyawen Hu \\ Department of Reproductive Immunology, Tongde Hospital of Zhejiang Province, Hangzhou, China \\ Contributions: (I) Conception and design: P Dong; (II) Administrative support: L Ling; (III) Provision of study materials or patients: L Hu; (IV) \\ Collection and assembly of data: L Ling; (V) Data analysis and interpretation: P Dong; (VI) Manuscript writing: All authors; (VII) Final approval of \\ manuscript: All authors. \\ Correspondence to: Lanyawen Hu. Department of Reproductive Immunology, Tongde Hospital of Zhejiang Province, Hangzhou 310012, China. \\ Email: vivian_hlyw@163.com.
}

Backgroundk To perform meta-analysis to investigate the efficacy and safety of traditional Chinese
medicine (TCM) compound in the treatment of endometriosis (EMS)-induced infertility.
Methods: The databases of PubMed, Embase, Cochrane Library, Chinese Journal Full-text Database
(CNKI), VIP, Wanfang Science and Technology Journal Full-text Database, and Chinese Biomedical
Literature (CBM) were used to search for articles on the treatment of EMS-induced infertility with TCM
compound from database establishment to September 2021 . Endnote X9 software was used to screen the
articles. Stata 15.1 and RevMan 5.3 software were used to record the data, and a meta-analysis was performed
on the effective rate, pregnancy rate, abortion rate, incidence of adverse reactions, luteinizing hormone (LH)
level, and estradiol (E2) level of TCM compound in the treatment of EMS-induced infertility. Finally, the
reliability of the results was assessed by sensitivity analysis. A funnel plot was used to evaluate the publication
bias of the articles.

Results: A total of 11 articles were included. Meta-analysis showed that when TCM compound was used to treat EMS-induced infertility, the effective rate of the trial group was significantly higher than that in the control group [odds ratio $(\mathrm{OR})=1.26$; $95 \%$ confidence interval (CI): 1.00 to $1.60 ; \mathrm{P}=0.049$ ], and the difference was statistically significant; the pregnancy rate of the trial group was significantly higher than that in the control group ( $\mathrm{OR}=1.94 ; 95 \% \mathrm{CI}: 1.50$ to $2.50 ; \mathrm{P}<0.05)$, and the difference was statistically significant; the abortion rate of the trial group was significantly lower than that in the control group ( $\mathrm{OR}=0.16 ; 95 \% \mathrm{CI}: 0.06$ to $0.48 ; \mathrm{P}=0.01$ ), and the difference was statistically significant; and the incidence of adverse reactions in the trial group was not significantly different from the control group $(\mathrm{OR}=0.48$; 95\% CI: 0.17 to $1.34 ; \mathrm{P}=0.162$ ); the $\mathrm{LH}$ level of the trial and control group [standardized mean difference $(\mathrm{SMD})=0.51 ; 95 \% \mathrm{CI}:-1.73$ to $2.75 ; \mathrm{P}=0.658$ ], and the $\mathrm{E} 2$ level $(\mathrm{SMD}=1.65 ; 95 \% \mathrm{CI}:-0.77$ to 4.07 ; $\mathrm{P}=0.182$ ) had no statistical difference.

Discussion: Chinese herbal compound is effective in the treatment of endometriotic infertility and has a positive effect on improving the pregnancy rate.

Keywords: Chinese herbal compound; endometriosis (EMS); infertility; meta-analysis

Submitted Oct 29, 2021. Accepted for publication Dec 16, 2021.

doi: 10.21037/apm-21-3425

View this article at: https://dx.doi.org/10.21037/apm-21-3425 


\section{Introduction}

Endometriosis (EMS) refers to the presence of endometrial tissue (glands and stroma) outside the uterine body (1). Its pathogenesis is not clear (2), but there is a consensus that EMS is an inflammatory disease, immune disease, bleeding disorder, and hormone-dependent disease (3). The main clinical manifestations are pelvic pain, infertility, and pelvic nodules and bullae (4). Early symptoms are not obvious, and it is easy to appear misdiagnosis and missed diagnosis. Attention should be paid to identification with malignant ovarian tumors, pelvic inflammatory cysts, and uterine adenomyomas, and conduct B-ultrasound and MRI examination when necessary. It has been reported that 30 $50 \%$ of EMS patients will be complicated with infertility (5). For women with EMS and infertility, the main purpose of drug therapy is to not only reduce their pain, but also to improve and promote fertility. This type of drug therapy is mostly used by young patients. Generally, the course of treatment is 6-9 months. Drug therapy for EMS falls under endocrine therapy, which can be divided into four major categories: combined oral contraceptive (COC) drugs, androgen derivatives, highly effective progesterone, and gonadotropin-releasing hormone agonist (GnRH-a). It can control the menstrual cycle, inhibit the ovulatory process, cause pseudopregnancy or amenorrhea, and achieve the atrophy of ectopic endometrium, thereby promoting the necrosis and absorption of the lesion (6). In addition, aromatase inhibitors and gonadotropinreleasing hormone antagonist (GnRH-ant) have also been used to some extent. Despite the existence of this treatment, the clinical effect is often unsatisfactory, gastrointestinal symptoms, abnormal uterine bleeding, perimenopausal symptoms, weight gain, and other side effects are obvious, and the recurrence rate remains high. As a branch of traditional Chinese medicine (TCM) therapy, research of TCM compound in the treatment of EMS is increasing annually, so it is necessary to strictly evaluate the efficacy of TCM compound in the treatment of EMS infertility, in order to provide a scientific basis for it in clinical application. There are many studies that have been conducted on EMS, and this meta-analysis aims to systematically evaluate the efficacy of TCM compound therapy in EMS through the inclusion of the latest studies. We present the following article in accordance with the PRISMA reporting checklist (available at https://dx.doi. org/10.21037/apm-21-3425).

\section{Methods}

\section{Criteria for inclusion of literature in the study}

\section{Literature type}

All included studies were randomized controlled trials (RCTs), the language was limited to Chinese and English, and other types of studies such as reviews, experience summaries, basic experiments, cohort studies, and casecontrol studies were excluded.

\section{Participants}

The participants included in the literature were women under 40 years of age, and studies conducted with animals such as rats and rabbits were excluded. All the selected participants had a definite diagnosis of endometriotic infertility.

\section{Description of intervention}

The articles included two treatment groups for EMSinduced infertility: the trial group used TCM compound combined with western medicine, the control group used western medicine treatment alone, and the treatment comprised a full course of treatment.

\section{Outcome indicators}

The primary outcome indicators included response rate and pregnancy rate.

The secondary outcome indicators included abortion rate, incidence of adverse effects, luteinizing hormone (LH) levels, and estradiol (E2) levels.

\section{Exclusion criteria}

Experience summaries, case or case studies, basic studies with rats, rabbits, and other animals, studies primarily investigating other diseases, both treatment and control group using TCM compound studies, and non-RCT studies were excluded from this meta-analysis.

\section{Search strategy and literature identification}

Studies related to the treatment of endometriotic infertility with TCM compound were searched for in the databases of PubMed, Embase, Cochrane Library, Chinese Journal Full-text Database (CNKI), VIP, Wanfang Science and Technology Journal Full-text Database, and Chinese 
Biomedical Literature (CBM) from database establishment to September 2021. The English library was searched by means of medical subheadings (MeSH) words combined with free words, and the English library search was performed using the keywords (Chinese traditional medicine OR Chinese herbal) AND (endometriosis OR chocolate cyst). The English library was replaced by PubMed: \#1: MeSH endometriosis; \#2: MeSH 'Chinese traditional'; \#3: Title/Abstract ('Chinese traditional medicine' OR 'Chinese herbal'); \#4: Title/Abstract (endometriosis OR 'chocolate cyst'); \#5: \#1 OR \#4; \#6: \#2 OR \#3; \#7: \#5 AND \#6.

\section{Literature screening and data extraction}

After the retrieval of literature was completed, Endnote X9 software (Clarivate Analytics, Philadelphia, PA, USA) was used for management. The software automatic duplicate checking function was used to locate and eliminate duplicate documents. The articles were independently screened by two reviewers. The title and abstract was read for preliminary screening, and the full text was downloaded for further review after excluding unqualified articles. When the two reviewer's opinions conflicted, disagreements were resolved through discussion with a 3 rd reviewer.

The two reviewers independently completed the data extraction, and the relevant contents were extracted were as follows:

(I) Basic information of the article: title, author, contact address, country, name of publication journal, publication time;

(II) Characteristics of the study: total sample size, number of groups, number of samples per group;

(III) Participant characteristics: age, symptom classification;

(IV) Characteristics of the intervention: different intervention methods used in the trial group and control group;

(V) Result assessment: the types and number of adverse reactions.

\section{Literature bias and evaluation analysis}

The assessment of bias was performed according to the Cochrane Collaboration's risk of bias tool (7). Assessments considered the generation of random sequences, allocation concealment, blinding of participants and implementers, blinding of outcome evaluators, selective reporting, completeness of outcome data, and other biases. Literature quality evaluation was completed independently by two reviewers, and when disagreement occurred, a 3 rd reviewer was invited to arbitrate to resolve the disagreement.

\section{Measurement of effect}

Binary variables (response rate, pregnancy rate, abortion rate, adverse effects) were assessed using odds ratio (OR) and their $95 \%$ confidence interval (CI); continuous variables (LH levels, E2 levels) were assessed using standardized mean difference (SMD) and their 95\% CI.

\section{Handling method for data loss}

In the case of unprovided data in the literature, if it could be obtained by calculation, it was obtained by calculation; if there was no data at all, the data was obtained by contacting the author, and if it was still not available, the article in question was excluded.

\section{Statistical analysis and heterogeneity detection}

The software Stata 15.1 (StataCorp. LLC, College Station, TX, USA) was used for analysis, and a forest plot was used to represent analysis results. The $\mathrm{I}^{2}$ and $\mathrm{Q}$ tests were used to analyze literature heterogeneity, and $\mathrm{I}^{2}>50 \%$ or $\mathrm{P}<0.05$ was used to indicate statistical difference in heterogeneity.

\section{Publication bias analysis}

The presence of publication bias was analyzed by funnel plot.

\section{Heterogeneity survey and sensitivity analysis}

Heterogeneity was investigated using L'Abbe plots provided by Stata 15.1 and sensitivity analysis was performed using the influence analysis tool.

\section{Results}

\section{Literature search results}

A total of 11,215 articles were initially found in this search. After de-duplication and screening, 11 articles were included in the meta-analysis. The literature screening 


\section{Identification of studies via databases and registers}

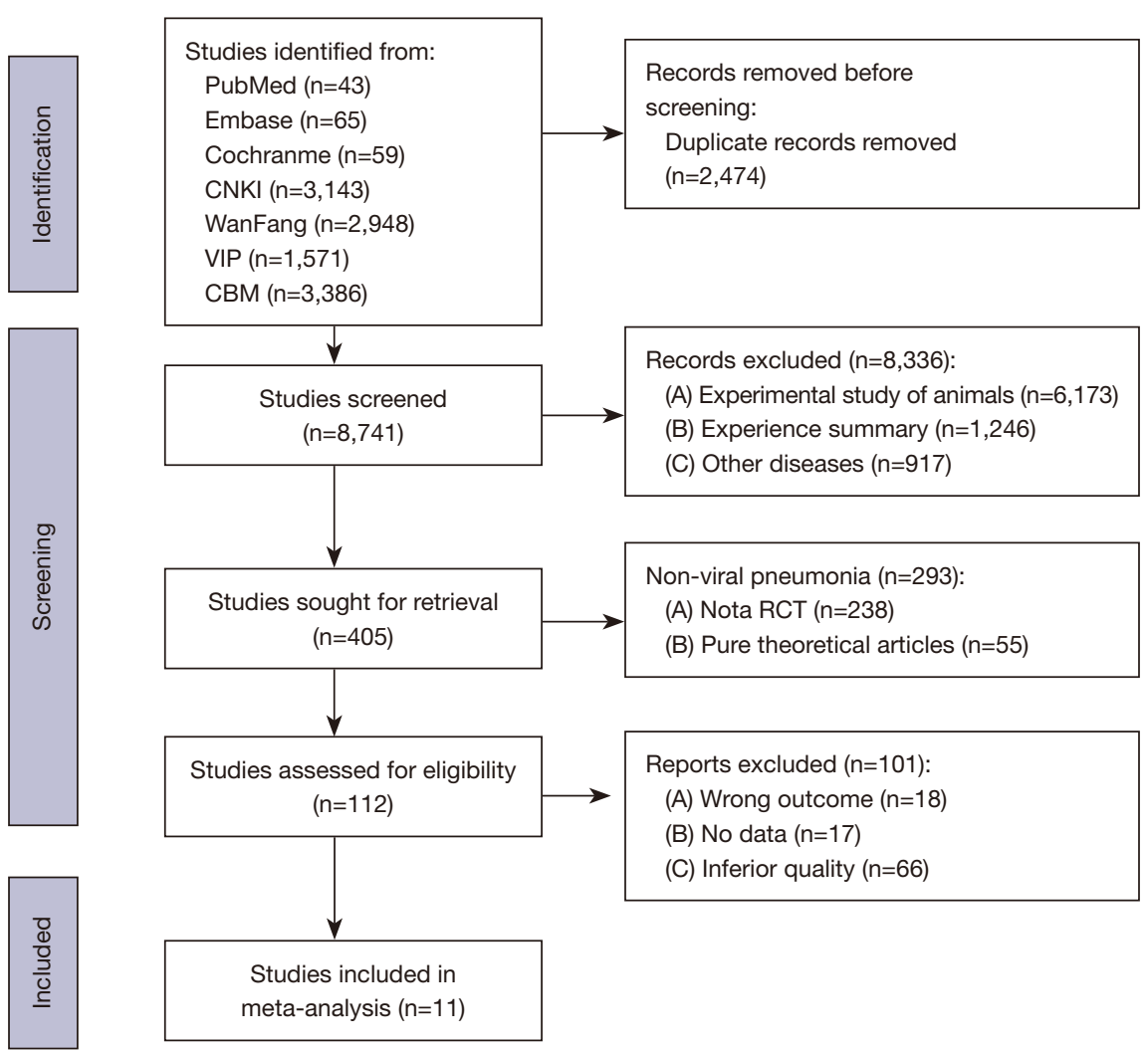

Figure 1 Literature screening flow chart. RCT, randomized controlled trial.

process and results are shown in Figure 1.

\section{Basic characteristics of included articles}

A total of 1,071 patients were included across the 11 articles in this study. Basic information of articles is shown in Table 1 .

\section{Quality assessment}

According to the Cochrane Collaboration's risk of bias tool, all included studies were low-risk studies, all articles used the random number table, all articles did not mention whether allocation concealment was performed, the blind method only mentioned the random number table, none mentioned whether there was a blind method for outcome evaluators, none mentioned whether there was an elective report, the outcome indicators were complete, and none contained other risks of bias. The risk of bias evaluation of the included studies is shown in Figures 2,3.

\section{Combined analysis of the results of the effective rate of TCM compound in the treatment of EMS-induced infertility}

A total of 7 articles $(8,13-18)$ mentioned the effective rate of TCM compound in treating EMS-induced infertility. Metaanalysis showed that the effective rate of the trial group was significantly higher than that in the control group $(\mathrm{OR}=1.26 ; 95 \% \mathrm{CI}: 1.00$ to $1.60 ; \mathrm{P}=0.049)$, and the difference was statistically significant, as shown in Figure 4.

\section{Combined analysis of the results of pregnancy rate of TCM compound in the treatment of EMS-induced infertility}

A total of 8 articles $(8-12,15,17,18)$ mentioned questions about the pregnancy rate of Chinese herbal compound in treating EMS-induced infertility. Meta-analysis showed that the pregnancy rate of the trial group was significantly higher than that in the control group $(\mathrm{OR}=1.94 ; 95 \% \mathrm{CI}$ : 


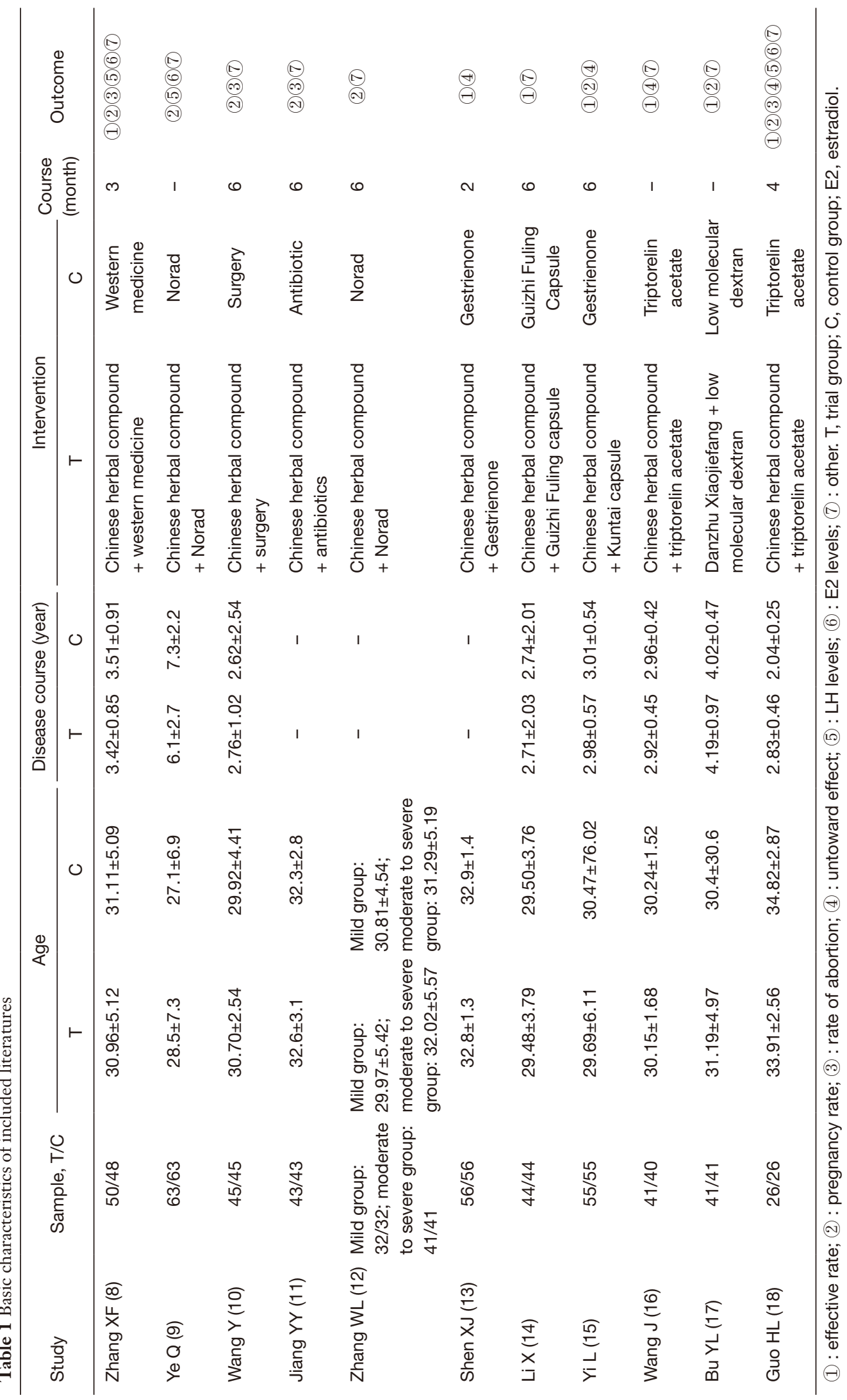




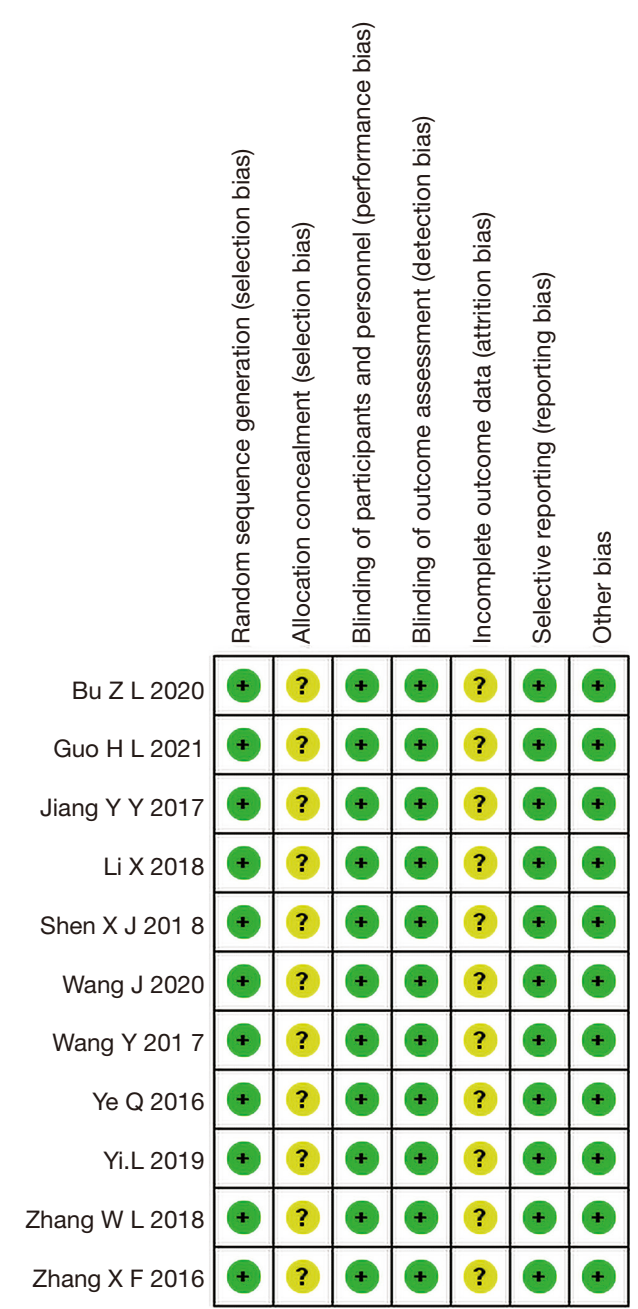

Figure 2 Risk summary of bias.
1.50 to $2.50 ; \mathrm{P}<0.05)$, and the difference was statistically significant, as shown in Figure 5.

Combined analysis of the results of abortion rate of TCM compound in the treatment of EMS-induced infertility

A total of 4 articles $(8,10,11,18)$ mentioned the issue of the abortion rate of TCM compound in the treatment of EMS-induced infertility. Meta-analysis showed that the abortion rate in the trial group was significantly lower than that in the control group (OR $=0.16 ; 95 \% \mathrm{CI}: 0.06$ to $0.48 ; \mathrm{P}=0.01$ ), with statistical significance, as shown in Figure 6.

\section{Analysis of adverse reactions of TCM compound in the treatment of EMS-induced infertility}

A total of 4 articles $(13,15,16,18)$ recorded the problems related to the adverse reactions of TCM compound in the treatment of EMS-induced infertility, and the meta-analysis results suggested that the incidence of adverse reactions in the trial group was not significantly different from that in the control group ( $\mathrm{OR}=0.48$; $95 \% \mathrm{CI}$ : 0.17 to 1.34 ; $\mathrm{P}=0.162)$, as shown in Figure 7.

Combined analysis of serum sex hormone levels in patients with EMS-induced infertility treated with Chinese herbal compound

A total of 3 articles $(8,9,18)$ mentioned the problems related

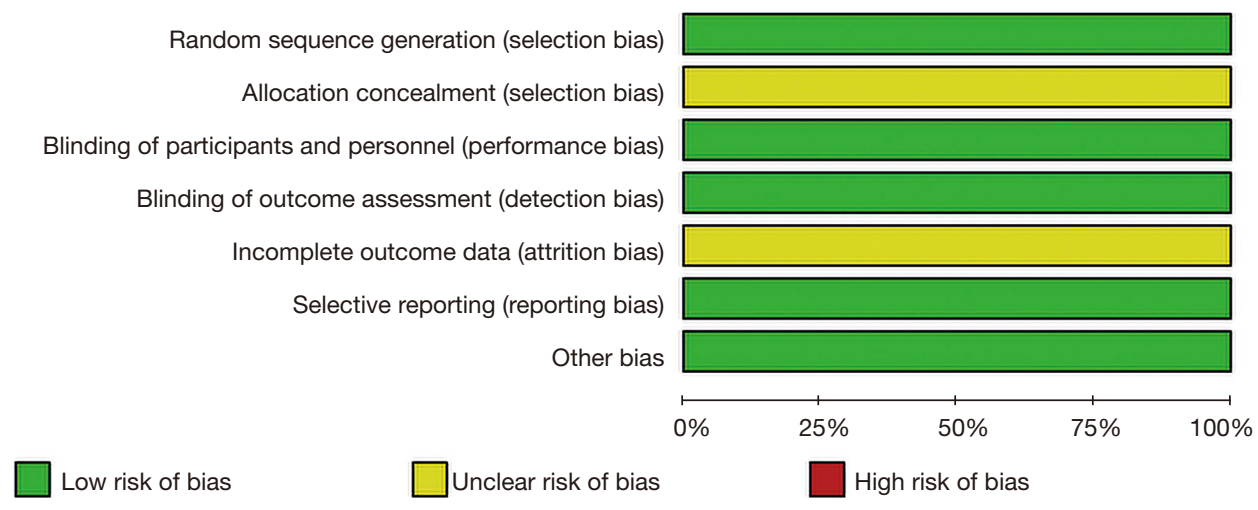

Figure 3 Risk of bias graph. 
Study

ID

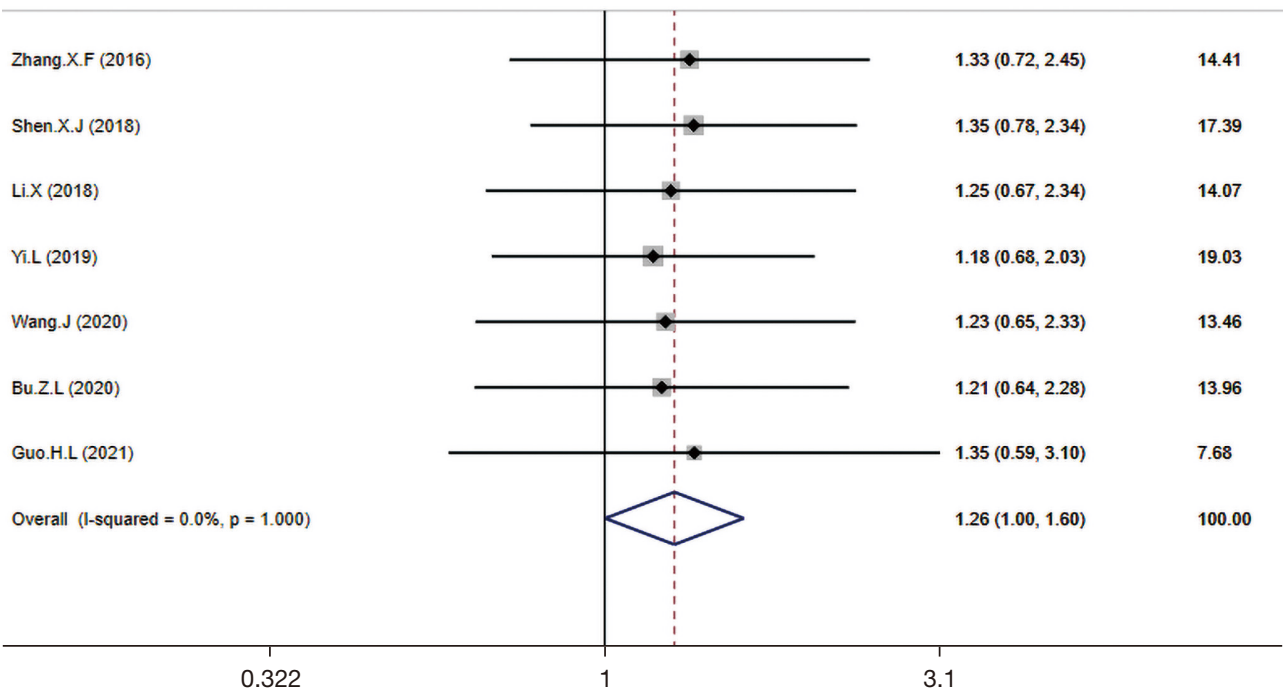

Figure 4 Forest plot of the effective rate of TCM compound in the treatment of EMS-induced infertility. TCM, traditional Chinese medicine; EMS, endometriosis; OR, odds ratio; CI, confidence interval.

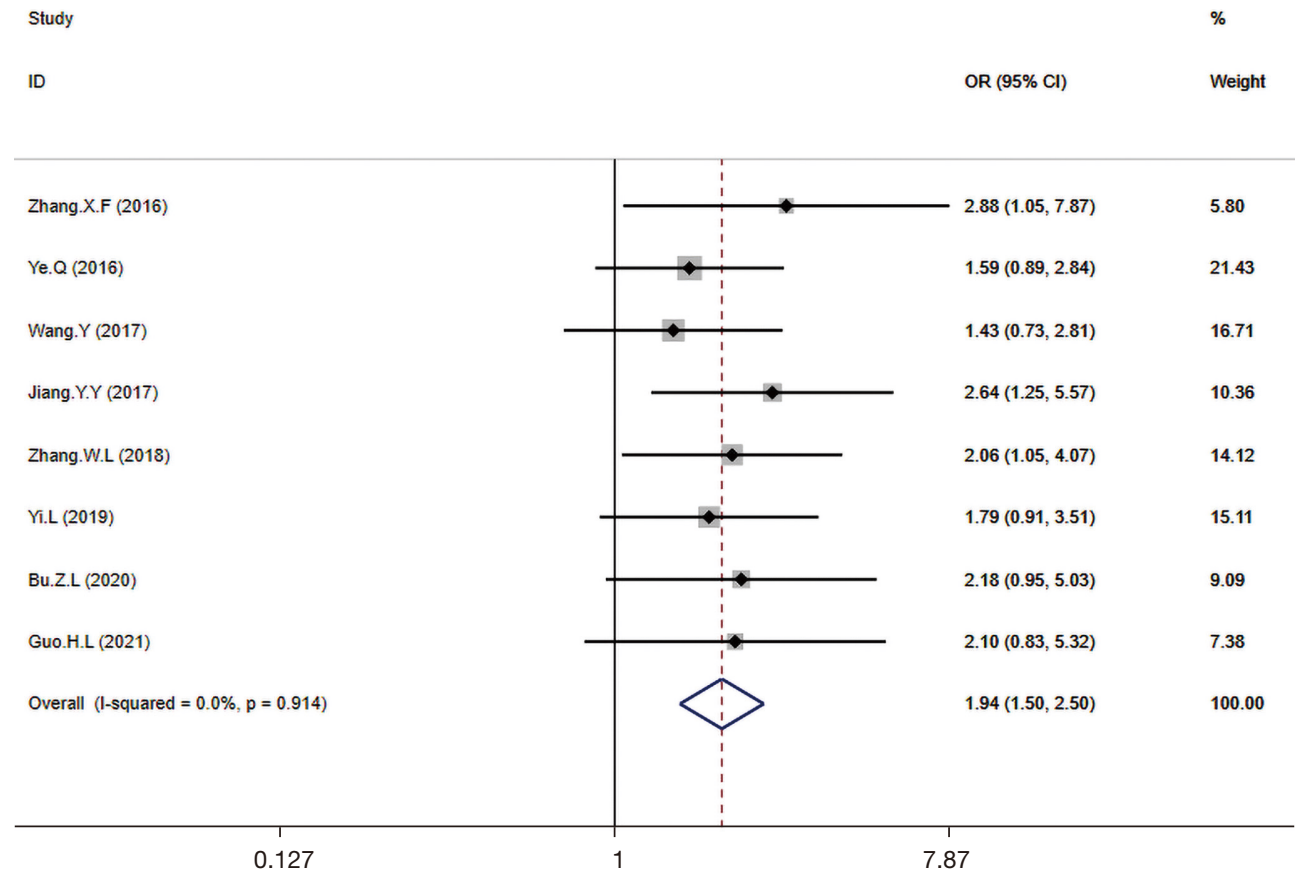

Figure 5 Forest map of the results of pregnancy rate of TCM compound in the treatment of EMS-induced infertility. TCM, traditional Chinese medicine; EMS, endometriosis; OR, odds ratio; CI, confidence interval. 


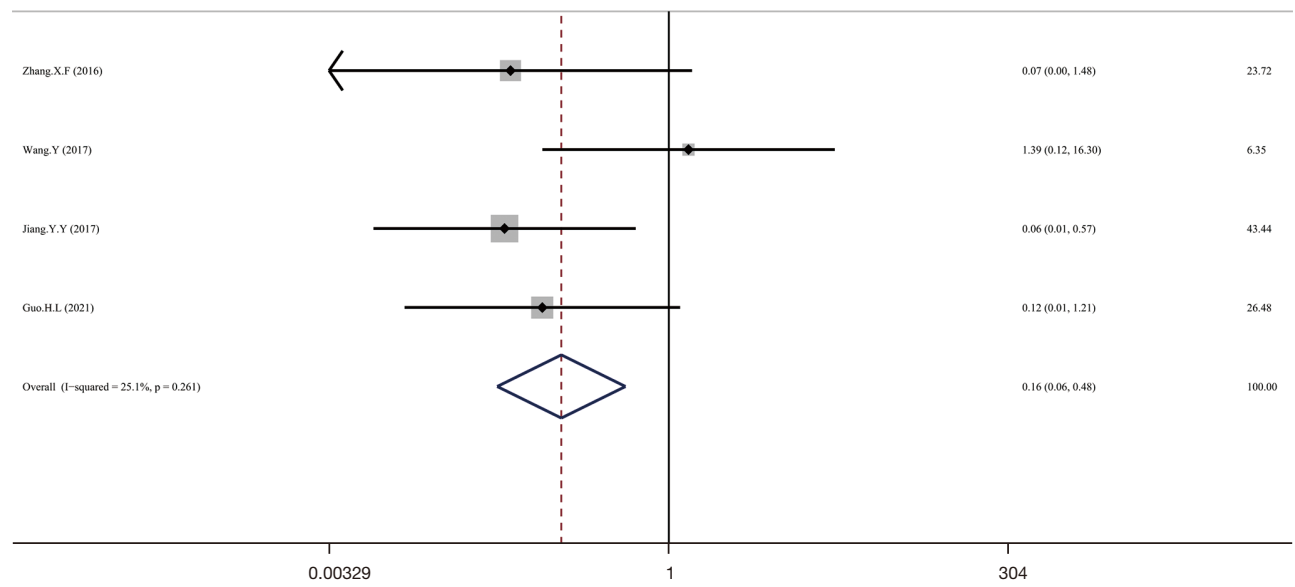

Figure 6 Forest map of the results of abortion rate of TCM compound in the treatment of EMS-induced infertility. TCM, traditional Chinese medicine; EMS, endometriosis; OR, odds ratio; CI, confidence interval.

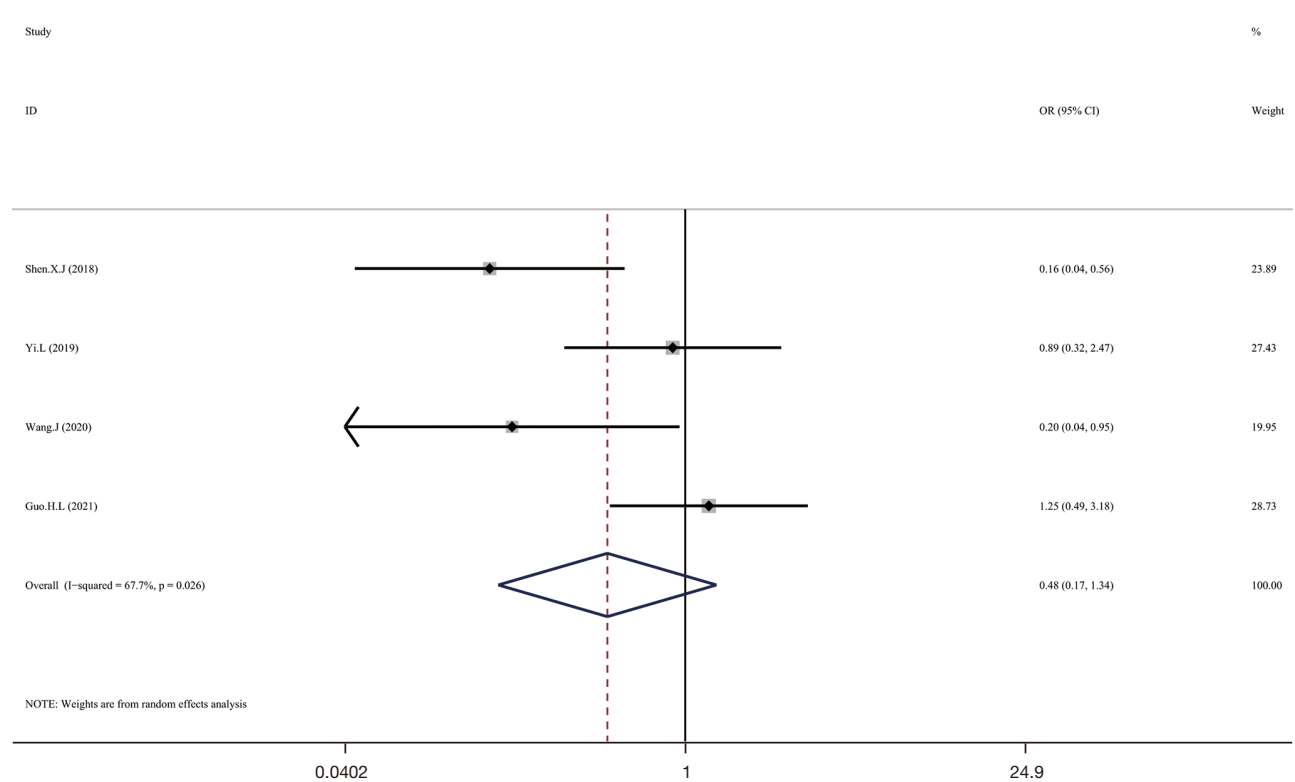

Figure 7 Forest map of the results of adverse reactions of TCM compound in the treatment of EMS-induced infertility. TCM, traditional Chinese medicine; EMS, endometriosis; OR, odds ratio; CI, confidence interval.

to the treatment of EMS infertility with TCM compound in terms of serum sex hormones. According to the results of meta-analysis, the LH level in the trial and control group (SMD $=0.51 ; 95 \%$ CI: -1.73 to $2.75 ; \mathrm{P}=0.658)$, and the $\mathrm{E} 2$ level (SMD $=1.65 ; 95 \%$ CI: -0.77 to $4.07 ; \mathrm{P}=0.182)$, with no statistical difference, as shown in Figures 8,9.

\section{Heterogeneity survey}

According to L'Abbe plot, this paper has less heterogeneity, 


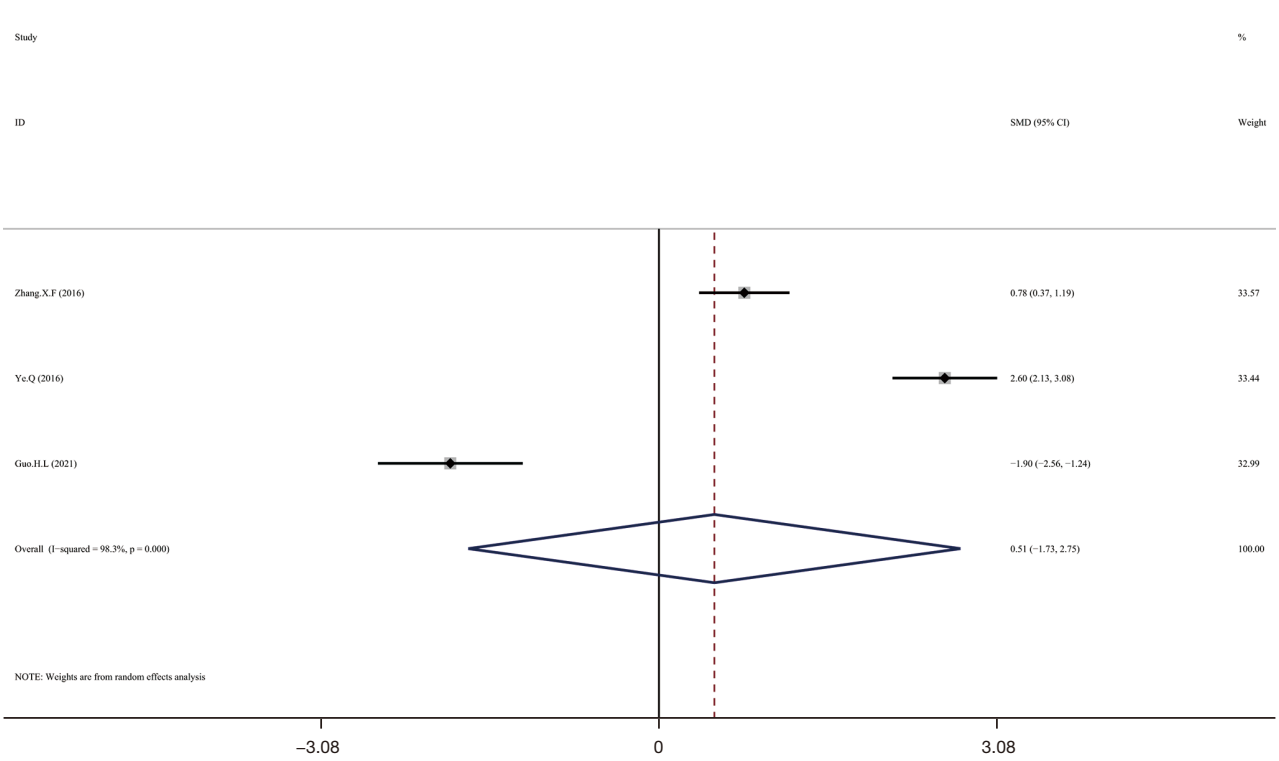

Figure 8 Combined forest plot of LH levels in patients with endometriotic infertility treated with TCM compound. LH, luteinizing hormone; TCM, traditional Chinese medicine; SMD, standardized mean difference; CI, confidence interval.

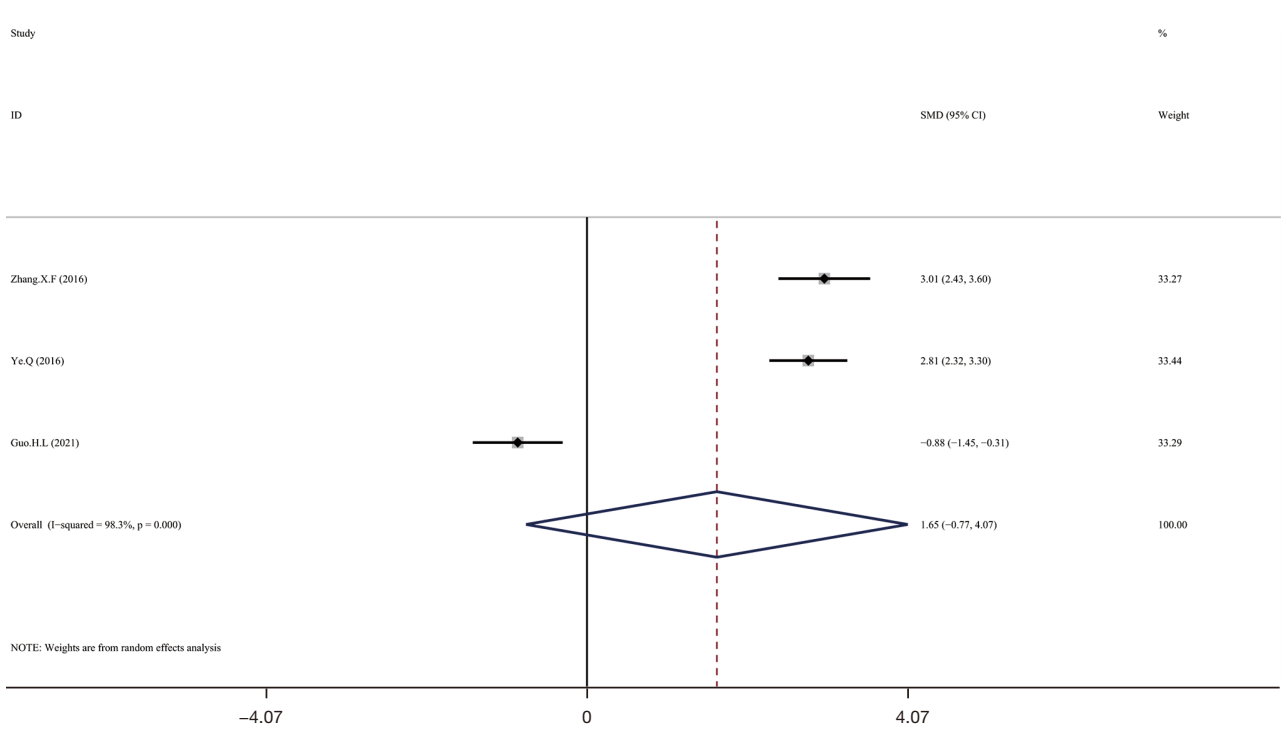

Figure 9 Forest map of the results of E2 level in the treatment of EMS-induced infertility with TCM compound. E2, estradiol; EMS, endometriosis; TCM, traditional Chinese medicine; SMD, standardized mean difference; CI, confidence interval.

as shown in Figure 10.

\section{Sensitivity analyses}

The sensitivity analysis of pregnancy rate showed that the two sides of the results distribution of the 8 articles were close and the stability was good (as shown in
Figure 11).

\section{Analysis of publication bias}

Funnel plots were made for the effective rate and pregnancy rate of the main outcome measures, and the results of the funnel plots showed that the left and right distribution of 


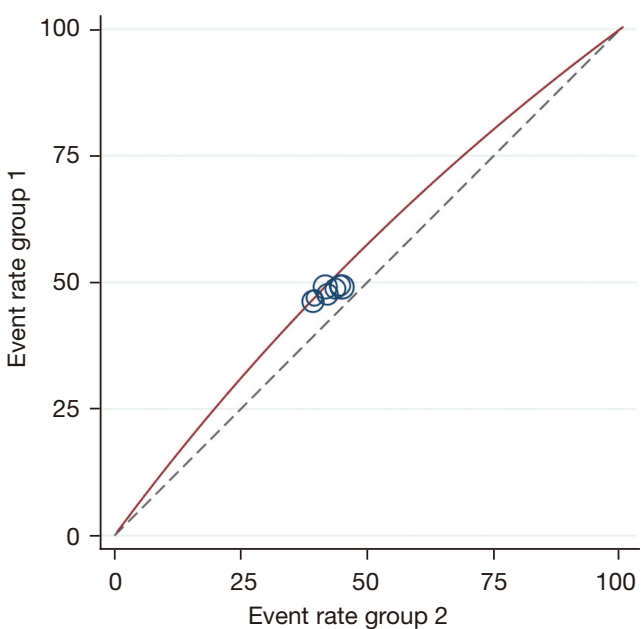

Figure 10 L'Abbe plot.

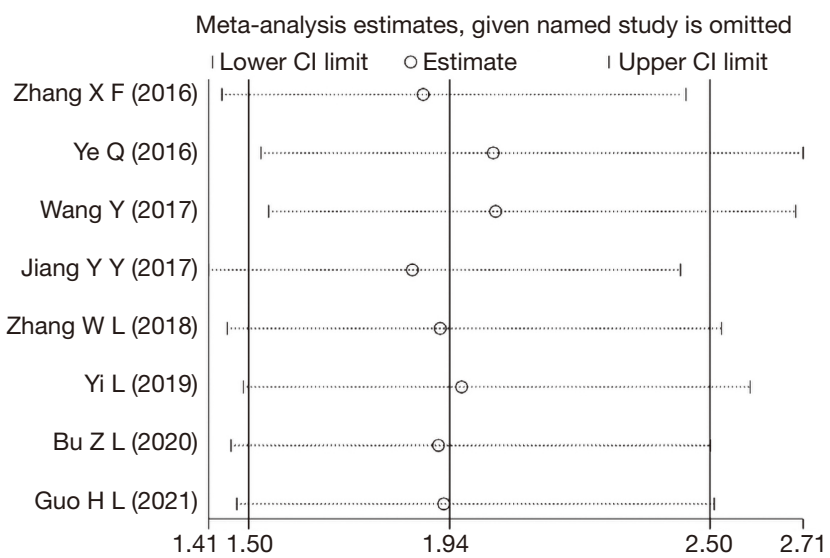

Figure 11 Sensitivity analysis of pregnancy rate. CI, confidence interval.

the literature was asymmetric, suggesting that there may have been publication bias (as shown in Figures 12,13).

\section{Discussion}

In this meta-analysis, a total of 11 articles were included, to a total sample size of 1,071 . Meta-analysis showed that when TCM compound was used to treat EMSinduced infertility, the effective rate of the trial group was significantly higher than that of the control group (OR $=1.26 ; 95 \%$ CI: 1.00 to $1.60 ; \mathrm{P}=0.049$ ), and the difference was statistically significant; the pregnancy rate of the trial group was significantly higher than that in the control group (OR $=1.94 ; 95 \% \mathrm{CI}: 1.50$ to $2.50 ; \mathrm{P}<0.05)$, and the

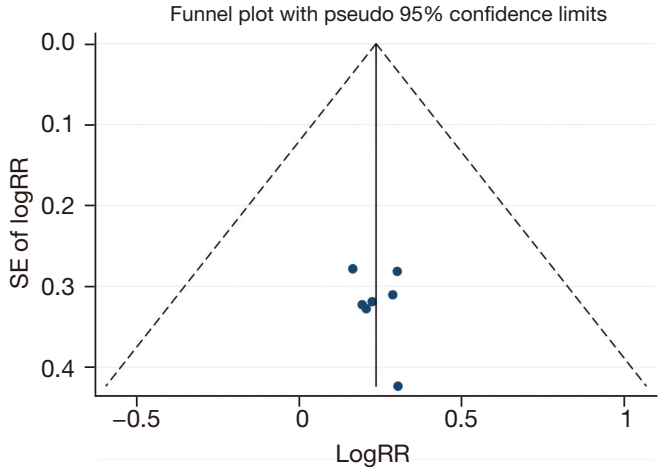

Figure 12 Funnel plot of effective rate. SE, standard error; RR, relative risk.

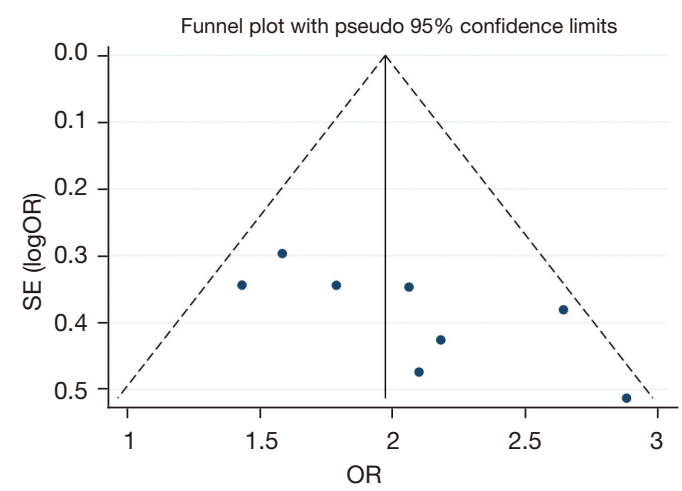

Figure 13 Funnel plot for pregnancy rate. SE, standard error; OR, odds ratio.

difference was statistically significant; the abortion rate of the trial group was significantly lower than that in the control group ( $\mathrm{OR}=0.16 ; 95 \% \mathrm{CI}$ : 0.06 to $0.48 ; \mathrm{P}=0.01$ ), and the incidence of adverse reactions in the trial group was not significantly different from that in the control group (OR $=0.48 ; 95 \%$ CI: 0.17 to $1.34 ; \mathrm{P}=0.162)$; the $\mathrm{LH}$ level of the trial and control group (SMD $=0.51 ; 95 \% \mathrm{CI}:-1.73$ to $2.75 ; \mathrm{P}=0.658)$, and the $\mathrm{E} 2$ level $(\mathrm{SMD}=1.65 ; 95 \% \mathrm{CI}$ : -0.77 to $4.07 ; \mathrm{P}=0.182)$ had no statistical difference. The results of qualitative analysis showed that most of the studies held a positive attitude towards the importance of efficacy and concluded that TCM compound could significantly improve the pregnancy rate of patients with EMS-related infertility. Regarding the studies of adverse reactions and serum sex hormones, less than 4 studies mentioned the relevant aspects. Since the number of included studies was too small and the differences were mainly from the same article (18), it may have been due to the different quality of 
each study, with a high possibility of bias. More high-quality literatures still need to be included to verify the adverse reactions and the problems related to serum sex hormones. The funnel plot showed left-right asymmetry, suggesting that there may have been publication bias, and the L'Abbe plot also showed that there may be some publication bias, but it could be seen from the sensitivity analysis that the existing publication bias did not affect the correctness of the conclusion. In summary, TCM compound is effective in the treatment of EMS-induced infertility.

The above conclusions were based on the 11 included articles, and in terms of methodological quality, most of the included studies had low risks, and although the quality of the articles was intentionally controlled at the time of inclusion, strong conclusions could not be drawn, mainly because of the following aspects. (I) The sample size of the included articles was generally small, in order to provide a better reference for the study results, the study can follow the randomized controlled clinical report to standardize life to estimate the sample size and make full use of limited resources to draw a positive conclusion within the scope of ethics. (II) All articles did not declare the blind method and allocation concealment, and the concealment of allocation scheme was often ignored by researchers. The study found that compared with the experiments with perfect allocation scheme, the efficacy of the experiments with imperfect allocation scheme concealment was often exaggerated by $30-41 \%$ (19). The 11 studies included in this meta-analysis did not carry out the concealment of relevant allocation regimens because of the preparation and administration methods of TCM compounds, which is one of the reasons for the general low quality of TCM-related research (17). (III) Blind method is to eliminate the subjective influence of participants and researchers on the test results. All 11 studies included in this analysis mentioned blind method. At present, researchers in China generally ignore the use of blind method, which has a great impact on the reliability of study results. (IV) In TCM syndrome differentiation and treatment, medication is added or subtracted according to the syndrome, and there is no uniform standard for prescription and dose, which leads to greater heterogeneity of randomized trials and poor literature quality (20).

\section{Conclusions}

In summary, TCM compound in the treatment of EMS infertility has a better effect on the total effective rate, pregnancy rate, and can effectively control the abortion rate. However, due to the limited conditions of this meta-analysis, only Chinese and English literatures were searched, which might have led to bias. Due to uneven quality of the included studies and small sample size, the possibility of bias could not be excluded. Therefore, larger samples, higher quality, multicenter RCTs are still needed to validate the above conclusions.

\section{Acknowledgments}

Funding: None.

\section{Footnote}

Reporting Checklist: The authors have completed the PRISMA reporting checklist. Available at https://dx.doi. org/10.21037/apm-21-3425

Conflicts of Interest: All authors have completed the ICMJE uniform disclosure form (available at https://dx.doi. org/10.21037/apm-21-3425). The authors have no conflicts of interest to declare.

Ethical Statement: The authors are accountable for all aspects of the work in ensuring that questions related to the accuracy or integrity of any part of the work are appropriately investigated and resolved.

Open Access Statement: This is an Open Access article distributed in accordance with the Creative Commons Attribution-NonCommercial-NoDerivs 4.0 International License (CC BY-NC-ND 4.0), which permits the noncommercial replication and distribution of the article with the strict proviso that no changes or edits are made and the original work is properly cited (including links to both the formal publication through the relevant DOI and the license). See: https://creativecommons.org/licenses/by-nc-nd/4.0/.

\section{References}

1. Kennedy S, Bergqvist A, Chapron C, et al. ESHRE guideline for the diagnosis and treatment of endometriosis. Hum Reprod 2005;20:2698-704.

2. Kovács Z, Glover L, Reidy F, et al. Novel diagnostic options for endometriosis - Based on the glycome and microbiome. J Adv Res 2021;33:167-81.

3. Zeng XH, Zhou M, Lu AM, et al. Relationship between endometriosis and RANTES genetic polymorphism. 
Chinese Journal of Birth Health \& Heredity 2012;20:34-5, 45.

4. Lang JH. History, status quo, and development of the understanding of endometriosis. Chinese Journal of Practical Gynecology and Obstetrics 2020;36:193-6.

5. Alkatout I, Mettler L, Beteta C, et al. Combined surgical and hormone therapy for endometriosis is the most effective treatment: prospective, randomized, controlled trial. J Minim Invasive Gynecol 2013;20:473-81.

6. Song JY, Sun ZG, Wang AJ, et al. Systematic Review of Compound Chinese Herbal Formula on Pregnancy Rate in Endometriosis-Associated Infertility. World Journal of Integrated Traditional and Western Medicine 2017;12:18-22.

7. Higgins JP, Altman DG, Gøtzsche PC, et al. The Cochrane Collaboration's tool for assessing risk of bias in randomised trials. BMJ 2011;343:d5928.

8. Zhang XF, Zhang YQ. Effect of nourishing yin and tonifying yang Chinese medicine on serum sex hormone levels and pregnancy outcome of patients with endometriosis combined infertility. Chinese Journal of Human Sexuality 2016;25:129-32.

9. Ye Q, Sun LL, He S. The clinical studies of kidney in traditional Chinese medicine's therapy combined laparoscopic surgery in treatment of infertility endometriosis. Chinese Journal of Human Sexuality 2016;25:139-43.

10. Wang Y, Pan LZ. Clinical observation of TCM staging therapy after mild endometriosis and infertility by laparoscopy. Guangxi Journal of Traditional Chinese Medicine 2017;40:32-4.

11. Jiang YY, Tang JL. Clinical Observation of Laparoscopic Surgery Combined with Chinese Medicine in Treating Endometriosis Merged Infertility. Journal of New Chinese Medicine 2017;49:109-11.

12. Zhang WL, Zhao SQ. Curative effect observation of

Cite this article as: Dong P, Ling L, Hu L. Systematic review and meta-analysis of traditional Chinese medicine compound in treating infertility caused by endometriosis. Ann Palliat Med 2021;10(12):12631-12642. doi: 10.21037/apm-21-3425
Zishen Liangxue Tiaozhou method in the treatment of endometriosis with infertility patients and effect of VEGF in serum. Chinese Journal of General Practice 2018;16:1897-900.

13. Shen XJ. Effect of TCM comprehensive treatment of endometriosis combined with infertility. Clinical Medicine 2018;38:98-9.

14. Li X, Zhou YY. Bushen Huoxue recipe on endometrial receptivity in patients with kidney deficiency and blood stasis type of endometriosis-induced infertility. Shaanxi Journal of Traditional Chinese Medicine 2018;39:341-4.

15. Yi L. Splknot analgesia soup combined with Kuntai capsule treated 55 cases of infertility caused by endometriosis. Traditional Chinese Medicinal Research 2019;32:23-6.

16. Wang J, Li YF. Clinical effects of TCM cycle therapy on endometriosis infertility. Medical Journal of Chinese People's Health 2020;32:105-7.

17. Bu YL. Clinical observation of Dan combined elimination with laparoscopy in the treatment of mild endometriosis infertility. Journal of Electrocardiogram (Electronic Edition) 2020;9:95-6.

18. Guo HL, Huang XQ, He Y. Effects of laparoscopic combined traditional Chinese and Western medicine in patients with endometriosis and infertility. Henan Medical Research 2021;30:2258-60.

19. Schulz KF, Chalmers I, Hayes RJ, et al. Empirical evidence of bias. Dimensions of methodological quality associated with estimates of treatment effects in controlled trials. JAMA 1995;273:408-12.

20. Zhou HY, Li WH, Tang DY, et al. Meta-analysis of endometriosis by promoting blood circulation and removing blood stasis. Journal of Practical Traditional Chinese Internal Medicine 2021;35:1-4, 143-144.

(English Language Editor: J. Jones) 